\title{
Kelvin-Helmholtz instability in MHD flows
}

\author{
A. H. Khater ${ }^{1,2}$, D. K. Callebaut ${ }^{2}$ and A. R. Seadawy ${ }^{1}$ \\ ${ }^{1}$ Department of Mathematics, Faculty of Science, Beni-Suef University, Beni-Suef, Egypt \\ ${ }^{2}$ Department Natuurkunde, CGB, University of Antwerp, B-2020 Antwerp, Belgium
}

\begin{abstract}
The Kelvin-Helmholtz instability (KHI) could be a potential source for some of the Alfvénic fluctuations observed in the solar wind. The nonlinear evolution of KHI is examined in $2+1$ dimensions in the context of magnetohydrodynamics. We derived a nonlinear Schrödinger equation (NLSE) and we obtained soliton solutions of this $2+1$ dimensions NLSE.
\end{abstract}

Keywords. MHD; Kelvin-Helmholtz instability ; solar wind; planetary interiors.

\section{Problem Formulation}

The KHI occurs in fluids and plasmas when shear in the unperturbed flows is presented across the interface between two different layers. This instability has been investigated in detail in various physical situations, such as the magnetospheric boundary layer, astrophysical jets, the solar wind, a dynamically evolving, turbulent MHD and laboratory plasmas (Chandrasekhar, 1961). Andries and Goossens studied the effect of the velocity shear between the coronal plume and the interplume region on the spectrum of MHD waves trapped in the plume. This type of instability may be excited at the boundary of the magnetosphere by the solar winds (Andries et al., 2000, Andries et al., 2001).

In this paper, we consider two-dimensional semi-infinite, incompressible fluids separated by the interface $z=0$, along the direction of streaming. The fluid of density $\rho_{1}$ with magnetic permeability $\mu_{1}$ occupies the half-space $z<0$, and the fluid of density $\rho_{2}$ with magnetic permeability $\mu_{2}$ is in the half-space $z>0$. The lower and the upper fluids are moving along the x-axis with uniform velocities $U^{(1)}$ and $U^{(2)}$. The system is under the influence of a gravitational force $\mathbf{g}(0,0,-\mathrm{g})$. The basic equations governing the velocity potential $\phi(v=\nabla \phi)$ and the magnetic potential $\psi(\mathbf{H}=-\nabla \psi)$ are

$$
\nabla^{2} \phi^{(1)}=\nabla^{2} \psi^{(1)}=0, \quad-\infty<z<\eta, \quad \nabla^{2} \phi^{(2)}=\nabla^{2} \psi^{(2)}=0, \quad \eta<z<\infty,
$$

with $\left|\nabla \phi^{(1)}\right| \rightarrow 0, \quad\left|\nabla \psi^{(1)}\right| \rightarrow 0 \quad$ as $z \rightarrow-\infty$, and $\quad\left|\nabla \phi^{(2)}\right| \rightarrow 0, \quad\left|\nabla \psi^{(2)}\right| \rightarrow 0$ as $z \rightarrow \infty$. The boundary conditions at the free interface $z=\eta(x, y, t)$, are given by

$$
\begin{gathered}
\partial \phi^{(j)} / \partial z-\partial \eta / \partial t-\left(U^{(j)} \cdot \nabla\right) \eta=\nabla \phi^{(j)} \cdot \nabla \eta, \quad \mu H_{n}^{(1)}=H_{n}^{(2)}, \quad H_{T}^{(1)}=H_{T}^{(2)}, \quad(1.2) \\
\rho_{1}\left(\frac{\partial \phi^{(1)}}{\partial t}+U^{(1)} \frac{\partial \phi^{(1)}}{\partial x}+U^{(1)} \frac{\partial \phi^{(1)}}{\partial y}\right)-\rho_{2}\left(\frac{\partial \phi^{(2)}}{\partial t}+U^{(2)} \frac{\partial \phi^{(2)}}{\partial x}+U^{(2)} \frac{\partial \phi^{(2)}}{\partial y}\right) \\
+\left(\rho_{1}-\rho_{2}\right) g \eta+\frac{1}{2} \rho_{1}\left(\nabla \phi^{(1)}\right)^{2}-\frac{1}{2} \rho_{2}\left(\nabla \phi^{(2)}\right)^{2}-T\left[\nabla^{2} \eta\left(1+(\nabla \eta)^{2}\right)-(1 / 2) \nabla \eta \cdot \nabla(\nabla \eta)^{2}\right] \\
\times\left(1+(\nabla \eta)^{2}\right)^{-2 / 3}=\left(\mu_{2}(\mu-1) / 8 \pi\right)\left(\left(H^{(1)}\right)^{2}+(\mu-1)\left(H_{n}^{(1)}\right)^{2}\right), j=1,2, \quad(1.3)
\end{gathered}
$$


where $\mu=\frac{\mu_{1}}{\mu 2}, H_{n}$ and $H_{T}$ represent the normal and tangential components of the magnetic field. Both magnetic fluids are assumed to be linearly magnetizable. The nonlinear stability problem posed by Eqs. (1.1) to (1.3) is examined.

\section{Linear and Nonlinear Analysis}

The method employed is that of multiple time and space scales [Nayfeh, 1981]. Linearized equations for the system are derived and the dispersion relation is obtained as

$$
D\left(\omega, k, l, U^{(j)}\right)=\frac{\rho_{j} \sigma_{j}^{2}}{\rho_{1}+\rho_{2}}-k-k^{3}-k l^{2}-(k+l)^{2}\left(\frac{H^{2}}{4 \pi \mu_{2}\left(\rho_{1}+\rho_{2}\right)}\right) \frac{(1-\mu)^{2}}{\mu+1},
$$

where $\sigma_{j}=(k+l) U^{(j)}-\omega$. The neutral point of the instability occurs at

$$
\left(U^{(1)}-U^{(2)}\right)^{2}=\frac{\left(\rho_{1}+\rho_{2}\right)^{2}}{\rho_{1} \rho_{2}}\left(\frac{k+k^{3}+k l^{2}}{(k+l)^{2}}+\left(\frac{H^{2}}{4 \rho\left(\rho_{1}+\rho_{2}\right)}\right) \frac{(1-\mu)^{2}}{\mu+1}\right) .
$$

It is obvious from (2.1) and (2.2) that the system is unstable for $U \geqslant U_{c}$ and the system is stable for $U<U_{c}$. From The second- and third-order theories, we have derived a NLSE for the wavenumbers, when the velocity difference between the lower and the upper fluid is less than the critical velocity $U_{c}$ as

$$
2 i \frac{\partial A}{\partial \tau}+\frac{\partial^{2} A}{\partial \xi_{1}^{2}}+\frac{\partial^{2} A}{\partial \eta_{1}^{2}}=Q|A|^{2} A
$$

where $\quad \xi_{1}=\frac{x_{1}}{P_{1}^{1 / 2}}, \quad \eta_{1}=\left(P_{3}-\frac{P_{2}^{2}}{P_{1}}\right)^{-1 / 2}\left(y_{1}-\frac{P_{2}}{P_{1}} x_{1}\right), \quad \tau=t_{2}$, and $Q ; P_{1} ; P_{2} ; P_{3}$ are constants. Eq. (2.3) have soliton solutions as (Khater et al. 2001, 2003)

$$
\begin{gathered}
A=\sqrt{2 C / Q} \sec \left(\sqrt{C}\left(d_{0} \tau+d_{1} \xi_{1}+d_{2} \eta_{1}\right)+d\right) \exp i\left(c_{0} \tau+c_{1} \xi_{1}+c_{2} \eta_{1}\right) \\
A=\sqrt{C / Q} \tanh \left(\sqrt{C / 2}\left(d_{0} \tau+d_{1} \xi_{1}+d_{2} \eta_{1}\right)+d\right) \exp i\left(c_{0} \tau+c_{1} \xi_{1}+c_{2} \eta_{1}\right)
\end{gathered}
$$

Where $C, d_{0}, d_{1}, d_{2}, c_{0}, c_{1}, c_{2}$ are real constants. The soliton stability of Eq. (2.3) can be obtained by deriving the functional relation $\delta L / \delta A^{*}=0$, from the Lagrangian

$$
L=\iint\left(\frac{i}{2}\left(A^{*} \frac{\partial A}{\partial \tau}-A \frac{\partial A^{*}}{\partial \tau}\right)-\frac{1}{2}\left(\left|\frac{\partial A}{\partial \xi_{1}}\right|^{2}+\left|\frac{\partial A}{\partial \eta_{1}}\right|^{2}+\frac{Q}{2}|A|^{2}\right)\right) d \xi_{1} d \eta_{1} .
$$

\section{Conclusion}

The nonlinear stage of the two-dimensional KHI is studied by including the effect of surface tension between the two fluids. By using the multiple scales method linearized equations for the system are derived and the dispersion relation is obtained. From the second- and third-order theories, we have derived a NLSE in $2+1$ dimensions, when the velocity difference between the lower and the upper fluid is less than the critical velocity $U_{c}$. We obtained the soliton solutions of $2+1$ dimensions NLSE. This allows to discuss the stability of the soliton solutions.

\section{References}

Andries, J., Tirry, W. J. \& Goossens, M. 2000, Astrophy. J. 531, 561. 
Andries, J. \& Goossens, M., 2001, Astronomy Astrophy, 368, 1083.

Chandrasekhar, S., 1961, Hydrodynamic and Hydromagnetic Stability (Clarendon Press)

Khater, A. H., Callebaut, D. K., Malfliet, W. \& Seadawy, A. R., 2001, Physica Scripta, 64, 533.

Khater, A. H., Callebaut, D. K. \& Seadawy, A. R., 2003, Physica Scripta, 67, 340.

Nayfeh, A. H., 1981, Introduction to perturbation techniques, (Wiley, New York.). 